\title{
Random matrix theory of the proximity effect in disordered wires
}

\author{
M. Titov and H. Schomerus \\ Max-Planck-Institut für Physik komplexer Systeme, Nöthnitzer Strasse 38, 01187 Dresden, Germany \\ (Received 7 August 2002; revised manuscript received 22 October 2002; published 16 January 2003)
}

\begin{abstract}
We study analytically the local density of states in a disordered normal-metal wire $(N)$ at ballistic distance to a superconductor $(S)$. Our calculation is based on a scattering-matrix approach, which concerns for wavefunction localization in the normal metal, and extends beyond the conventional semiclassical theory based on Usadel and Eilenberger equations. We also analyze how a finite transparency of the NS interface modifies the spectral proximity effect and demonstrate that our results agree in the dirty diffusive limit with those obtained from the Usadel equation.
\end{abstract}

DOI: 10.1103/PhysRevB.67.024410

\section{INTRODUCTION}

It is widely acknowledged that a piece of a normal metal that is in good contact with a superconductor acquires some superconducting properties. This phenomenon, named the proximity effect, has already been studied by Cooper ${ }^{1}$ in the early 1960's. Since then many theoretical and experimental investigations have been carried out. ${ }^{2}$ Much owed to the recent progress in the fabrication technology of nanostructures there is a revived interest to the proximity effect in the last decade. ${ }^{3}$ One remarkable evidence of this effect is the formation of a spectral gap in the normal metal, which strongly affects the low-temperature transport properties of the normal-metal-superconductor $(N S)$ junctions. The key mechanism responsible for the appearance of the gap is the Andreev reflection at the $N S$ boundary, which converts the dissipative electrical current into dissipationless supercurrent. ${ }^{4}$ Similar mechanisms act in superconductor ferromagnet junctions which have become an object of intense study recently.,

An effective experimental technique which allows for spatially resolved measurements of the electronic density in the nanostructures is the scanning tunnelling microscopy. It provides both a unique sub-meV energy sensitivity and an atomic spatial resolution. Several recent measurements of the local electronic density of states (LDOS) in the $N S$ junctions ${ }^{10,11,8,7,9}$ turned out to be in very good agreement with the predictions of quasiclassical theory ${ }^{12-17}$ of "nonequilibrium" superconductivity, based on the Usadel equation for the diffusive transport ${ }^{18}$ and the Eilenberger equation for the ballistic transport. ${ }^{9}$

The interplay of ballistic and diffusive transport becomes important when one studies local properties at short distance to an NS interface in a disordered system. Quasiparticles are then transferred to the interface by ballistic transport, while they explore the rest of the system diffusively. This situation is not covered by conventional quasiclassical theory. Quasiclassics also cannot account for the nonperturbative effects of wave-function localization, which only can be included by a fully phase-coherent approach. In this paper we present a theory that goes beyond the quasiclassical description and apply it to calculate the local density of states in an NS wire geometry near the interface, at zero temperature and vanishing magnetic field.

In our model the normal metal is shaped in the form of the
PACS number(s): 74.50.+r, 72.15.Rn, 73.20.Fz, 74.45.+c

long disordered quantum wire, which supports $N$ propagating modes at the Fermi level $E_{F}$. The elastic scattering mean free path $l$ in the wire is assumed to be much larger than the Fermi wave length $\lambda_{F}$, which corresponds to the weak disorder. The superconductor is assumed to be clean and characterized by the bulk value $\Delta$ of the amplitude of the pair potential. The superconductor order parameter is assumed to be constant $\Delta$ in the superconductor and zero in the normal metal. This approximation is referred to in the literature as a "rigid boundary condition." 20

We calculate the mean LDOS or, more precisely, its envelope, at a distance $x$ on the normal-metal side of the $N S$ interface as shown schematically in Fig. 1. The envelope is obtained by averaging the LDOS over distances of the order of the Fermi wave length $\lambda_{F}$. The spatial averaging smears out the Friedel type oscillations and makes the LDOS independent on the position across the wire.

We study in detail the case that the distance $x$ is small compared to the scattering mean free path $l$, so that $\lambda_{F} \ll x$ $\ll l$, while the ratio between the superconductor coherence length $\xi=\hbar v_{F} / \Delta$ and $l$ remains arbitrary. The resulting mean LDOS found by averaging over disorder does not depend on $x$ and is a smooth function of energy everywhere except at $\varepsilon=\Delta$ (the energy $\varepsilon$ is measured from the Fermi surface).

Our calculation is organized as follows. In Sec. II we derive a general relation between the one-point Green func-

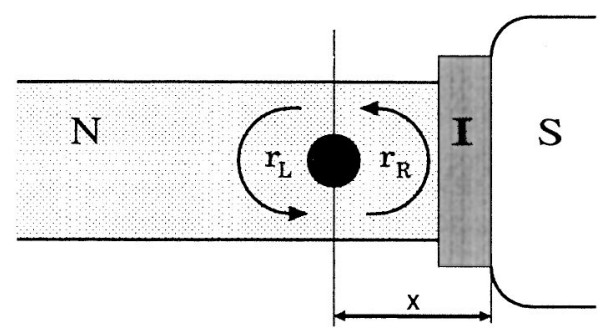

FIG. 1. The geometry of an NS junction consisting of a long normal-metal disordered wire $N$, a clean superconductor $S$, and a dielectric tunnel barrier $I$ in between. The mean local density of states (LDOS) is calculated at the distance $x$ from the $N S$ interface, with $\lambda_{F} \ll x \ll l$. The matrix $r_{L}$ relates the plane-wave components in the process of reflection from the normal-metal disordered wire. The matrix $r_{R}$ describes the reflection from the tunnel-barriersuperconductor part of the junction. The mean LDOS is found by averaging over the disorder-induced fluctuations of the matrix $r_{L}$. 

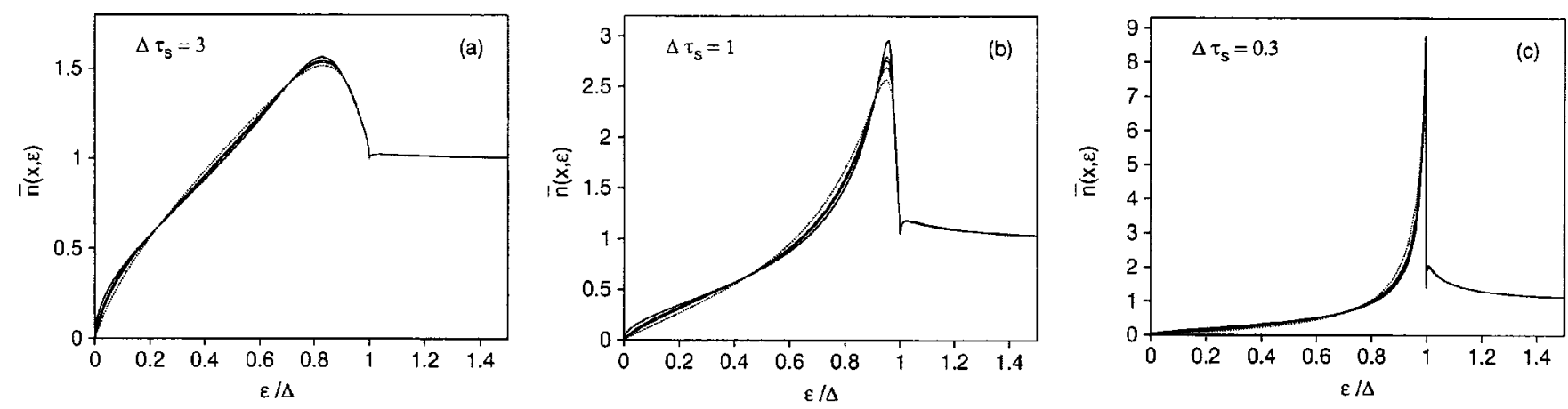

FIG. 2. The mean LDOS (25) in an $N$-channel normal-metal wire near an ideally transmitting $N S$ interface. The curves are calculated from Eqs. (24),(25). The thick solid (dotted) line corresponds to the limiting case of the multi(single)-channel wire. The thin lines are for the finite number of channels $N=2,3$, and 4 . The figures correspond to the clean regime $\Delta \tau_{s}=3$, the intermediate regime $\Delta \tau_{s}=1$, and the dirty regime $\Delta \tau_{s}=0.3$.

tion in a quantum wire and the reflection matrices $r_{L}, r_{R}$. These matrices relate the plane-wave components of the quasiparticle wave function in the process of reflection from the parts of the wire to the left and to the right part of $x$.

We apply this result in Sec. III in order to calculate the mean LDOS in the neighborhood of an ideally transmitting NS interface. The matrices $r_{L}, r_{R}$ of the size $2 N \times 2 N$ describe the reflection of the electronlike and holelike quasiparticles. The left reflection matrix $r_{L}$ is diagonal in the electron-hole representation and depends on the disorder in the normal metal. The right reflection matrix $r_{R}$ is offdiagonal (in absence of the tunnel barrier) and is fixed within the model considered. LDOS

In the region $\varepsilon<\Delta$ we obtain the disorder-averaged

$$
\bar{n}(x, \varepsilon)=\pi \rho_{\varepsilon}\left(\phi_{A}\right), \quad \phi_{A}=\arccos \varepsilon / \Delta,
$$

where the function $\rho_{\varepsilon}(\phi)$ is the probability density of the eigenphase of the matrix correlator $r_{0}(\varepsilon) r_{0}(-\varepsilon)^{\dagger}$. The reflection matrix $r_{0}(\varepsilon)$ relates the plane-wave amplitudes of the electron wave function in the process of reflection from the semi-infinite normal-metal wire. The probability density $\rho_{\varepsilon}(\phi)$ has been studied in Ref. 21. Apart from energy and phase it depends on the number of channels $N$ and the mean scattering time $\tau_{s}=l / v_{F}$. According to Ref. 21 one can distinguish localized, diffusive and ballistic regimes in the form of the function $\rho_{\varepsilon}(\phi)$ depending on the value of $\varepsilon$. We observe the effect of Anderson localization in the linear increase of the LDOS for energies smaller than the Thouless energy $\varepsilon_{c}=\hbar / N^{2} \tau_{s}$. We also find that the curves calculated for different number of channels in the wire are lying close to each other at any ratio $l / \xi$ (see Fig. 2). This suggests that the weak-localization correction to the LDOS is small in the case of the ideally transmitting NS interface.

In Sec. IV we generalize the model to include a tunnel barrier at the interface, parametrized by a tunnel probability per mode $\Gamma$. We calculate analytically the LDOS near the interface in the extreme cases of a localized wire $N=1$ and a diffusive wire $N \gg 1$.

The effect of the tunnel barrier consists of a reduction of the pseudogap in the normal metal. This effect is most pro- nounced in the dirty regime $l \leq \xi$ or $\Delta \tau_{s} / \hbar \leqslant 1$. The results of our calculation for the diffusive wire in the intermediate regime $l=\xi$ are summarized in Fig. 3 for different values of $\Gamma$. We observe that the LDOS increases monotonously to its bulk constant value around the energy $\hbar \Gamma^{2} / \tau_{s}$ and reveals a high and narrow peak close to $\varepsilon=\Delta$.

The monotonous reduction of the pseudogap is attributed to the quasiparticles which experience normal reflection at the tunnel barrier and therefore do not see the NS boundary. The formation of the peak is due to the quasiparticles reflected from the superconductor.

When the distance $x$ increases beyond the mean free path $l$ a competing effect takes place. That is the suppression of the pseudogap due to the back scattering on the weak disorder potential in the normal-metal segment of length $x$ in front of the interface. The estimated size of the pseudogap due to this effect is $\hbar D / x^{2}$, where $D$ is the diffusion constant in the normal metal. In this case the LDOS considerably overshoots its bulk value around $\varepsilon=\hbar D / x^{2}$, which is in contrast to the monotonous increase due to the tunneling into the superconductor. We therefore anticipate that the effect of the tunnel barrier still can be seen in the shape of the LDOS provided $\hbar D / x^{2} \gg \hbar \Gamma^{2} / \tau_{s}$, or equivalently $x \ll l / \Gamma$. Namely, at dis-

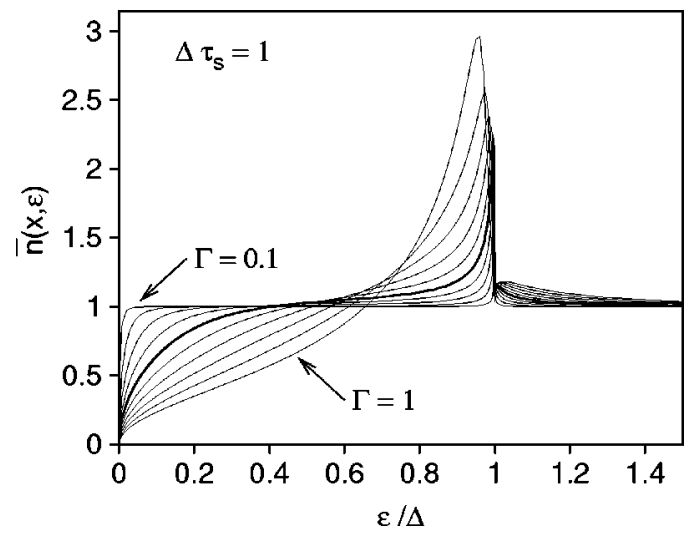

FIG. 3. The mean LDOS in a diffusive normal-metal wire in the vicinity of an $N S$ interface of finite transparency. The curves are calculated from Eqs. (54),(57) for $\Delta \tau_{s}=1$ and the effective tunneling probability $\Gamma$ varying from 0.1 to 1 in steps of 0.1 . The thick line corresponds to $\Gamma=0.5$. 
tances smaller than $l / \Gamma$ the LDOS may acquire the steplike feature at the value $\varepsilon=\hbar \Gamma^{2} / \tau_{s}$, which is fixed by the NS interface transparency rather than by the distance to the interface.

A qualitatively similar phenomenon has been indeed observed in experiments by Levi et al. ${ }^{9}$ in the $\mathrm{Cu}$ barrier pin wires near a $N(\mathrm{Cu})-S(\mathrm{NbTi})$ boundary.

On the contrary, at large distances $x \gg l / \Gamma$ the barrier is not effective in the sense that its presence cannot be distinguished in the energy dependence of the LDOS. This is consistent with a general semiclassical criterion ${ }^{22}$ which states that the barrier is not effective for a given observable if the most of the relevant trajectories hit the $N S$ interface more than $\Gamma^{-1}$ times before the electron-hole coherence is lost. In the case of the LDOS this criterion is fulfilled for $x \gg l / \Gamma$.

The tunnel barrier acts differently for the single-channel wire. In the dirty regime $l \leq \xi$ the size of the pseudogap $\hbar \Gamma / \tau_{s}$ scales linearly with $\Gamma$ due to the Anderson localization. This results in a different shape of the LDOS compared to the diffusive case $(N \gg 1)$. The difference becomes more and more pronounced with decreasing ratio $l / \xi$ or tunneling probability $\Gamma$. In Sec. V we compare the LDOS for the diffusive case $(N \gg 1)$ found from our theory to the LDOS calculated from the Usadel equation. ${ }^{14}$

\section{GREEN FUNCTION IN A WIRE GEOMETRY}

In our model of the NS junction the normal metal is shaped in the form of a semi-infinite quasi-one-dimensional disordered wire. The properties of such a system is well understood in the framework of the scattering theory ${ }^{23}$ provided the weak disorder limit $\lambda_{F} \ll l$. The detailed statistical description of the disorder scattering is based on the DorokhovMello-Pereyra-Kumar (DMPK) equation. ${ }^{24,25}$ This is a scaling equation for the probability distribution of the scattering matrix of a segment of the wire. Below we derive a general relation between the one-point Green function and the reflection matrices $r_{L}, r_{R}$ for two parts of the wire. The singlechannel counterpart of this relation has been used recently to reconsider the problem of LDOS fluctuations in onedimensional (1D) normal-metal wires. ${ }^{26}$

The disordered wire has the Hamiltonian $H=H_{0}+V(\vec{r})$, where $V(\vec{r})$ is a disordered potential. We parametrize $\vec{r}$ $=(x, \vec{\rho})$, where $x$ is the coordinate along the wire and $\vec{\rho}$ is the vector in the transversal direction. We first discuss the case of "spinless" electrons, assuming $H_{0}=-\left(1 / 2 m_{e}\right) \nabla^{2}$, $\hbar=1$, and include holelike quasiparticles in Secs. III and IV.

In the absence of $V$ the quantization in the transversal direction gives rise to a set of $N$ propagating modes characterized by the transverse momentum $\vec{q}_{n}$. The total energy $E=\left(1 / 2 m_{e}\right)\left(\left|\vec{q}_{n}\right|^{2}+k_{n}^{2}\right)$, where the $x$ momentum $k_{n}$ is conserved. The retarded Green function $G^{R}(E)=(E+i \eta$ $-H)^{-1}$ is written in the channel representation as

$$
G_{n m}^{R}\left(x, x^{\prime}\right)=\iint_{A} d \vec{\rho} d \vec{\rho}^{\prime}\langle n \mid \vec{\rho}\rangle\left\langle\vec{\rho}^{\prime} \mid m\right\rangle\left\langle\vec{r}\left|G^{R}\right| \vec{r}^{\prime}\right\rangle,
$$

where the integration is carried out over a cross-sectional area $A$. Hence the LDOS

$$
n(\vec{r}, \varepsilon)=-\frac{1}{\pi} \operatorname{Im} \sum_{n, m}\langle m \mid \vec{\rho}\rangle\langle\vec{\rho} \mid n\rangle G_{n m}^{R}(x, x),
$$

where $\varepsilon$ is the energy measured from the Fermi surface. For a two-dimensional wire of the width $d$ we have $\langle\rho \mid n\rangle$ $=(2 / d)^{1 / 2} \sin (\pi n \rho / d)$. In what follows we shall omit the in$\operatorname{dex} R$, assuming everywhere the retarded Green function.

Let us formally cut the wire in the point $x$ into two pieces and treat the left and the right part separately. We decompose the potential $V=V_{R}+V_{L}$, where $V_{R, L}$ is the disorder potential in the right and the left part of the wire, respectively. We also introduce the left and the right Green function as $G_{R, L}$ $=\left(E+i \eta-H_{0}-V_{L, R}\right)^{-1}$. According to Fisher and Lee (Ref. 27) we have

$$
G_{L, R ; n m}(x, x)=\frac{1}{i \sqrt{v_{n} v_{m}}}\left[\delta_{n m}+r_{L, R ; n m}(x)\right],
$$

where $v_{n}=k_{n} / m_{e}$ is the channel velocity and $r_{L, R}$ are the reflection matrices from the left and the right part of the wire, respectively.

The Green functions obey Dyson equations which can be written in the matrix form as

$$
\begin{aligned}
& \hat{G}(x, x)=\hat{G}_{0}(x, x)+\int_{-\infty}^{\infty} d y \hat{G}_{0}(x, y) \hat{V}(y) \hat{G}(y, x), \\
& \hat{G}(x, x)=\hat{G}_{R}(x, x)+\int_{-\infty}^{x} d y \hat{G}_{R}(x, y) \hat{V}_{L}(y) \hat{G}(y, x), \\
& \hat{G}(x, x)=\hat{G}_{L}(x, x)+\int_{x}^{\infty} d y \hat{G}_{L}(x, y) \hat{V}_{R}(y) \hat{G}(y, x),
\end{aligned}
$$

where the elements of the matrix $\hat{V}$ are given by

$$
V_{n m}(x)=\int_{A} d \vec{\rho}\langle n \mid \vec{\rho}\rangle\langle\vec{\rho} \mid m\rangle V(\vec{r}),
$$

and the ballistic Green function (in absence of the potential) reads

$$
G_{0, n m}\left(x, x^{\prime}\right)=\frac{\delta_{n m}}{i v_{n}} e^{i k_{n}\left|x-x^{\prime}\right|} .
$$

We also take advantage of the following relations: ${ }^{28}$

$$
\begin{aligned}
& G_{R, n l}(x, y)=e^{-i k_{l}(x-y)} G_{R, n l}(x, x) \text { for } y<x, \\
& G_{L, n l}(x, y)=e^{-i k_{l}(x-y)} G_{L, n l}(x, x), \quad \text { for } y>x,
\end{aligned}
$$

in the disorder-free regions in order to eliminate the integral terms in Eq. (5). As a result we obtain the matrix equality

$$
\frac{1}{\hat{G}(x, x)}+\frac{1}{\hat{G}_{0}(x, x)}=\frac{1}{\hat{G}_{R}(x, x)}+\frac{1}{\hat{G}_{L}(x, x)} .
$$


Using Eqs. (4) and (7) we finally get

$$
\hat{G}(x, x)=\frac{1}{\sqrt{i \hat{\mathrm{v}}}}\left(1+\hat{r}_{R}\right) \frac{1}{1-\hat{r}_{L} \hat{r}_{R}}\left(1+\hat{r}_{L}\right) \frac{1}{\sqrt{i \hat{\mathrm{V}}}},
$$

where $\hat{\mathrm{v}}$ is the diagonal matrix of channel velocities $v_{n}$. Together with Eq. (3) this equation defines the LDOS via the reflection matrices. In the case of uncorrelated disorder the reflection matrices $r_{L}$ and $r_{R}$ are statistically independent, which makes Eq. (10) useful for practical calculations.

In general the LDOS oscillates on the scale of $\lambda_{F}$ (due to the prevailing contribution of one particular quantum state). These Friedel-type oscillations can play a crucial role especially in one dimension. In what follows we are concerned with the smoothed version of the LDOS that does not change on the scale of the Fermi wave length and, therefore, also not in the transversal direction. For this purpose we introduce the spatially averaged LDOS

$$
n(x, \varepsilon)=\delta V^{-1} \int_{\delta V} n(\vec{r}, \varepsilon) d \vec{r},
$$

where the integration is carried out over a volume $\delta V$ around the point $(x, \vec{\rho})$. The linear size of the volume $\delta V$ is assumed to be much larger than the Fermi wave length and much smaller than the mean free path $l$. For $\left|x-x^{\prime}\right| \ll l$ the reflection matrices defined at the cross section $x^{\prime}$ are related to those defined at $x$ by

$$
\begin{gathered}
r_{L}\left(x^{\prime}\right)=e^{-i \hat{k}\left(x-x^{\prime}\right)} r_{L}(x) e^{-i \hat{k}\left(x-x^{\prime}\right)}, \\
r_{R}\left(x^{\prime}\right)=e^{i \hat{k}\left(x-x^{\prime}\right)} r_{R}(x) e^{i \hat{k}\left(x-x^{\prime}\right)},
\end{gathered}
$$

with $\hat{k}=m_{e} \hat{\mathrm{v}}$. Expanding the right-hand side of Eq. (10) in a geometric series in $r_{L}, r_{R}$ we notice that only the terms with equal numbers of $r_{L}$ and $r_{R}$ matrices do not oscillate on the scale of the Fermi wave length and have to be kept. Additionally the averaging in the transversal direction mixes up the different modes so that $\langle m \mid \vec{\rho}\rangle\langle\vec{\rho} \mid n\rangle \propto \delta_{m n}$ in Eq. (3). As a result we obtain

$$
n(x, \varepsilon)=\frac{n_{0}}{N} \operatorname{Re} \operatorname{Tr} \frac{1+r_{R} r_{L}}{1-r_{R} r_{L}},
$$

where $n_{0}$ is the bulk value of the LDOS in the normal metal, which is set to unity in the rest of the paper. In what follows we apply Eq. (13) to calculate the LDOS in the normal-metal wire in the immediate vicinity of an $N S$ interface.

\section{LDOS NEAR THE IDEAL NS INTERFACE}

The relation (13) applies straightforwardly to the model of the $N S$ junction discussed in the Introduction. The only modification is the doubling of size of the reflection matrices due to particle-hole conversion. We still denote the number of electron channels in the wire by $N$, so that the size of the particle-hole reflection matrix is now $2 N$. Equation (13) can be written in the form

$$
n(x, \varepsilon)=1+\frac{2}{2 N} \operatorname{Re} \operatorname{Tr} \sum_{n=1}^{\infty}\left(r_{L} r_{R}\right)^{n},
$$

where $r_{L}$ is the electron-hole reflection matrix for the long normal-metal wire, while $r_{R}$ is that for the ideal $N S$ interface. These reflection matrices are conveniently parametrized by

$$
r_{L}=\left(\begin{array}{cc}
r_{0}(\varepsilon) & 0 \\
0 & r_{0}(-\varepsilon)^{*}
\end{array}\right), \quad r_{R}=e^{-i \phi_{A}}\left(\begin{array}{ll}
0 & 1 \\
1 & 0
\end{array}\right),
$$

where $\phi_{A}=\arccos \varepsilon / \Delta$ is the Andreev phase and $r_{0}(\varepsilon)$ $\times\left[r_{0}(\varepsilon)^{*}\right]$ is $N \times N$ reflection matrix of the electronlike [holelike] quasiparticles for the normal-metal wire. The matrix product $r_{L} r_{R}$ is block off-diagonal, hence only the even powers $n$ contribute to the trace in Eq. (14). From Eqs. (14),(15) we obtain

$$
n(x, \varepsilon)=1+\frac{2}{N} \operatorname{Re} \operatorname{Tr} \sum_{n=1}^{\infty}\left[r_{0}(\varepsilon) r_{0}(-\varepsilon)^{*}\right]^{n} e^{-2 i n \phi_{A} .}
$$

The right-hand side of Eq. (16) is completely determined by the eigenvalues of the correlator $r_{0}(\varepsilon) r_{0}(-\varepsilon)^{*}$, which is a unitary matrix. Its eigenvalues are conveniently parametrized by $\exp \left(2 i \phi_{j}\right), j=1,2, \ldots, N$, where the phases $\phi_{j}$ are restricted to the interval $(0, \pi)$. The joint probability density $P_{\varepsilon}\left(\phi_{1}, \phi_{2}, \ldots, \phi_{N}\right)$ is a symmetric function with respect to any permutation of its arguments because of the statistical equivalence of the channels. This function has been studied in detail in Ref. 21. Our calculation is restricted to the mean LDOS $\bar{n}(x, \varepsilon) \equiv\langle n(x, \varepsilon)\rangle$, where the angular brackets correspond to the average over the disorder potential in the wire. In order to perform the average in Eq. (16), it is enough to know only the probability density $\rho_{\varepsilon}(\phi)$ of a single eigenphase. It is instructive to compare Eq. (16) with the similar representation of the integrated density of states in the case of the normal-metal wire of finite length, which has been analyzed recently. ${ }^{29}$

When the Andreev phase $\phi_{A}$ is real, i.e., for $\varepsilon<\Delta$, the mean LDOS is found from Eq. (16) as

$$
\bar{n}(x, \varepsilon)=\pi \rho_{\varepsilon}\left(\phi_{A}\right), \quad \varepsilon<\Delta,
$$

where the eigenphase density $\rho_{\varepsilon}(\phi)$ is assumed to be normalized to unity on the interval $(0, \pi)$. The probability density $\rho_{\varepsilon}(\phi)$ acquires its simplest form in the case $N \gg 1$ of a large number of channels ${ }^{21}$

$$
\rho_{\varepsilon}(\phi)=\frac{1}{\pi \sin ^{2} \phi} \operatorname{Im} \sqrt{\left(\varepsilon \tau_{s}\right)^{2}+i \varepsilon \tau_{s}\left(1-e^{-2 i \phi}\right)}
$$

and in the single-channel case $\mathrm{c}^{30,31}$

$$
\rho_{\varepsilon}(\phi)=\frac{\varepsilon \tau_{s}}{\pi} \int_{0}^{\infty} \frac{\exp \left(-\varepsilon \tau_{s} t\right)}{t^{2} \sin ^{2} \phi-t \sin 2 \phi+1} d t .
$$

The scattering time $\tau_{s}$ of the DMPK scaling equation differs by a numerical factor (dependent on the dimensionality $d$ of the Fermi surface) from the mean scattering time of the 
transport theory $\tau_{s}^{\prime}$. Namely, $\tau_{s}=c_{d} \tau_{s}^{\prime}$, where $c_{d}$ $=2, \pi^{2} / 4,8 / 3$, for the dimensionality $d=1,2,3$, correspondingly.

Note, that the integrated density of states (DOS) $\nu$ $=L^{-1} \int_{0}^{L} \bar{n}(x, \varepsilon) d x$ in the infinite disordered wire $L \rightarrow \infty$ is given by the relation ${ }^{32} \nu=\pi(\partial / \partial \varepsilon)\left[\varepsilon \rho_{\varepsilon}(0)\right]$, which is similar in spirit to Eq. (17). For wires with on-site disorder (in standard universality classes) the value of $\pi \rho_{\varepsilon}(0)$ equals to unity irrespective of energy $\varepsilon$; however it can have a singularity at $\varepsilon=0$ for wires with a specific disorder symmetry.

So far we were only concerned with the mean LDOS for $\varepsilon<\Delta$. However, the result (17) can be easily extended to the energies above the pair potential value with the help of the analytical continuation $\varepsilon=i \omega$. On the other hand the analytical continuation has another crucial advantage. It transforms the dynamical correlator $r_{0}(\varepsilon) r_{0}(-\varepsilon)^{*}$ into the essentially static object $r_{0}(i \omega) r_{0}(i \omega)^{*}$. In the absence of a magnetic field the time-reversal symmetry is preserved and the reflection matrix $r_{0}$ is symmetric, hence $r_{0}(i \omega)^{*}=r_{0}(i \omega)^{\dagger}$. The eigenvalues $\exp \left(2 i \phi_{j}\right)$ of the matrix $r_{0}(\varepsilon) r_{0}(-\varepsilon)^{*}$ are transformed to the real eigenvalues $R_{j}$ of the matrix $r_{0}(i \omega) r_{0}(i \omega)^{*}$, which are the probabilities of the reflection from the long disordered wire in the presence of a spatially uniform fictitious absorption $\omega$.

The summation in Eq. (14) is performed in terms of the eigenvalues

$$
n(x, \varepsilon)=\left.\frac{1}{N} \operatorname{Re} \sum_{j=1}^{N} \frac{1-R_{j} \alpha^{2}(\omega)}{1+R_{j} \alpha^{2}(\omega)}\right|_{\omega=-i \varepsilon+0^{+}},
$$

where $0^{+}$is an infinitesimally small positive imaginary part of energy which ensures the retarded Green function required in Eq. (3). We have also introduced

$$
\alpha(\omega)=i e^{-i \phi_{A}}=\sqrt{1+(\omega / \Delta)^{2}}-\omega / \Delta .
$$

The joint probability density of the eigenvalues $R_{j}$ for the infinitely long wire is given by the stationary solution of the DMPK equation. In the parametrization

$$
R_{j}=\frac{\sigma_{j}}{\sigma_{j}+2(N+1) \omega \tau_{s}}, \quad \sigma_{j} \in(0, \infty)
$$

this solution takes the simple form

$$
P\left(\left\{\sigma_{j}\right\}\right)=c_{N} \prod_{j=1}^{N} e^{-\sigma_{j} / 4} \prod_{k>j}\left|\sigma_{k}-\sigma_{j}\right|
$$

which we recognize as the orthogonal Laguerre ensemble of random matrix theory ${ }^{33}$ (with normalization constant $c_{N}$ ). This ensemble corresponds to the class $C I$ in the classification scheme of Ref. 34 . The probability density (one-point function) $\rho(\sigma)$, normalized to unity in the interval $(0, \infty)$, is given by ${ }^{35}$

$$
\begin{aligned}
\rho(\sigma)= & \frac{e^{-\sigma}}{N}\left(\sum_{n=0}^{N-1}\left[L_{n}^{(0)}(\sigma)\right]^{2}-\frac{1}{2} L_{N-1}^{(0)}(\sigma) L_{N-1}^{(1)}(\sigma)\right. \\
& \left.+\frac{1}{4} L_{N-1}^{(1)}(\sigma) \int_{0}^{\sigma} d \zeta e^{(\sigma-\zeta) / 2} L_{N-1}^{(0)}(\zeta)\right),
\end{aligned}
$$

where $L_{n}^{(p)}(\sigma)$ is the generalized Laguerre polynomial.

We substitute the parametrization (22) in Eq. (20) and average over disorder with the help of the density $P(\{\sigma\})$. The result reads

$$
\bar{n}(x, \varepsilon)=\left.\operatorname{Re} \int_{0}^{\infty} d \sigma \rho(\sigma) \frac{1-\frac{\alpha^{2}(\omega)-1}{2(N+1) \omega \tau_{s}} \sigma}{1+\frac{\alpha^{2}(\omega)+1}{2(N+1) \omega \tau_{s}} \sigma}\right|_{\omega=-i \varepsilon+0^{+}} .
$$

This equation extends Eq. (17) to energies larger than $\Delta$. It can also be applied for arbitrary $N$. In the large- $N$ limit the distribution $\rho(\sigma)$ can be approximated by ${ }^{35}$

$$
\lim _{N \rightarrow \infty} N \rho(\zeta N)=\frac{1}{2 \pi} \sqrt{\frac{4}{\zeta}-1}, \quad 0<\zeta<4 .
$$

Substituting this expression into Eq. (25) we reproduce the results of Eqs. (17),(18) for $\varepsilon<\Delta$. In the limit $\varepsilon \rightarrow 0$ this leads to the square root behavior of the $\operatorname{LDOS} \bar{n}(x, \varepsilon \rightarrow 0)$ $=\operatorname{Re} \sqrt{-i \varepsilon \tau_{s}}$. In the extremely dirty regime $\Delta \tau_{s} \rightarrow 0$ we reproduce the result of the conventional BCS theory

$$
\bar{n}(x, \varepsilon)=\operatorname{Re} \varepsilon / \sqrt{\varepsilon^{2}-\Delta^{2}} .
$$

The Thouless energy $\varepsilon_{c}=1 / N^{2} \tau_{s}$, however, remains unresolved within the multichannel approximation (26). In order to fix the scale $\varepsilon_{c}$ one has to take advantage of another limiting relation ${ }^{36}$

$$
\begin{aligned}
\lim _{N \rightarrow \infty} \rho(\zeta / N)= & J_{1}^{2}(2 \sqrt{\zeta})-J_{0}(2 \sqrt{\zeta}) J_{2}(2 \sqrt{\zeta}) \\
& +(2 \sqrt{\zeta})^{-1} J_{0}(2 \sqrt{\zeta}) J_{1}(2 \sqrt{\zeta}),
\end{aligned}
$$

where $J_{n}(z)$ are Bessel functions. In the limit $\varepsilon \rightarrow 0$ one can safely put $\alpha(\omega)=1$ in Eq. (25) and take the real part explicitly,

$$
\bar{n}(x, \varepsilon)=\int_{0}^{\infty} d \sigma \rho(\sigma) \frac{1}{1+\sigma^{2}\left[(N+1) \varepsilon \tau_{s}\right]^{-1}} .
$$

To leading order in $\varepsilon / \varepsilon_{c}$ the function $\rho(\sigma)$ in Eq. (29) can be approximated by its value at the origin $\rho(0)=1 / 2$, which holds for $\sigma \ll N^{-1}$ [see Eq. (28)]. We therefore obtain

$$
\bar{n}(x, \varepsilon)=\frac{\pi(N+1)}{4} \varepsilon \tau_{s} \approx \frac{\pi}{4 N}\left(\varepsilon / \varepsilon_{c}\right), \quad \varepsilon \ll \varepsilon_{c} .
$$

The factor $1 / N$ in the last expression reflects the fact that only a single channel is responsible for the nonvanishing LDOS at energies lower than $\varepsilon_{c}$. 
In Fig. 2 we plot the mean LDOS given by Eq. (25) against the ratio $\varepsilon / \Delta$ for different numbers of channels in the moderately dirty regime $\Delta \tau_{s}=0.3$, the intermediate regime $\Delta \tau_{s}=1$, and the moderately clean regime $\Delta \tau_{s}=3$. We observe that the curves are lying close to each other in all cases. (This suggests that the LDOS near the ideally transmitting interface is quite insensitive to phase-coherent effects.) The situation changes in the case of a finite transparency $\Gamma<1$ of the $N S$ interface.

\section{EFFECT OF A TUNNEL BARRIER}

\section{A. Model}

We now introduce the simplest model of a dielectric tunnel barrier at the ideal $N S$ interface. The mean LDOS is calculated in the normal-metal at a ballistic distance $x \ll l$ from the interface (see Fig. 1).

We describe the segment $I$ of the wire between the chosen cross section and the ideal NS interface (this segment includes the tunnel barrier) by its $S$ matrix

$$
S_{I}=\left(\begin{array}{cc}
r_{1}^{I} & t_{2}^{I} \\
t_{1}^{I} & r_{2}^{I}
\end{array}\right),
$$

where each block itself consists of block-diagonal matrices in the particle-hole representation

$$
\begin{aligned}
& r_{1,2}^{I}=\left(\begin{array}{cc}
r_{1,2}(\varepsilon) & 0 \\
0 & r_{1,2}(-\varepsilon)^{*}
\end{array}\right), \\
& t_{1,2}^{I}=\left(\begin{array}{cc}
t_{1,2}(\varepsilon) & 0 \\
0 & t_{1,2}(-\varepsilon)^{*}
\end{array}\right),
\end{aligned}
$$

and the matrices $r_{1,2}(\varepsilon), t_{1,2}(\varepsilon)$ are $N \times N$ electron reflection and transmission matrices corresponding to the segment $I$.

The right matrix $r_{R}$ in the fundamental formula (14) depends on the $S$ matrix of the segment $I$ [see Eqs. (31),(32)] and on the scattering matrix for Andreev reflection [see Eq. (15)]. A straightforward algebraic calculation gives ${ }^{23}$

$$
\begin{gathered}
r_{R}=\left(\begin{array}{cc}
r_{c}(\varepsilon) & -t_{c}(-\varepsilon)^{*} \\
t_{c}(\varepsilon) & r_{c}(-\varepsilon)^{*}
\end{array}\right) \\
t_{c}(\varepsilon)=e^{-i \phi_{A}(\varepsilon)} t_{2}(-\varepsilon)^{*} M(\varepsilon) t_{1}(\varepsilon), \\
r_{c}(\varepsilon)=r_{1}(\varepsilon)+e^{-2 i \phi_{A}(\varepsilon)} t_{2}(\varepsilon) r_{2}(-\varepsilon)^{*} M(\varepsilon) t_{1}(\varepsilon) \\
M(\varepsilon)=\left[1-e^{-2 i \phi_{A}(\varepsilon)} r_{2}(\varepsilon) r_{2}(-\varepsilon)^{*}\right]^{-1} .
\end{gathered}
$$

In general, if the segment $I$ contains some weak disorder (which is the case, for example, for $x>l$ ) the correlations between the matrices $r_{1,2}$ and $t_{1,2}$ for electronlike and holelike quasiparticles are nontrivial. We consider here the case that the segment $I$ contains no disorder, but a sufficiently steep tunnel barrier which makes no difference in the tunneling probability of electrons and holes. In this case we can omit the energy dependence in the matrices $r_{1,2}$ and $t_{1,2}$. In what follows we take advantage of the polar decomposition

$$
\left(\begin{array}{ll}
r_{1} & t_{2} \\
t_{1} & r_{2}
\end{array}\right)=\left(\begin{array}{cc}
u_{I} & 0 \\
0 & v_{I}^{T}
\end{array}\right)\left(\begin{array}{cc}
\sqrt{1-\Gamma} & i \sqrt{\Gamma} \\
i \sqrt{\Gamma} & \sqrt{1-\Gamma}
\end{array}\right)\left(\begin{array}{cc}
u_{I}^{T} & 0 \\
0 & v_{I}
\end{array}\right),
$$

where $u_{I}, v_{I}$ are some unitary matrices, which depend on a particular realization of the barrier, and $\Gamma$ is the diagonal matrix of the tunneling probabilities $\Gamma_{j}$. Time-reversal symmetry in the segment $I$ is assumed. Once the dependence on energy in the matrices $u_{I}, v_{I}$ and $\Gamma$ is disregarded we obtain from Eqs. (33),(34) the right reflection matrix

$$
\begin{gathered}
r_{R}=\left(\begin{array}{cc}
u_{I} & 0 \\
0 & u_{I}^{*}
\end{array}\right)\left(\begin{array}{cc}
e^{i \chi} \cos \theta & -i e^{i \chi} \sin \theta \\
-i e^{i \chi} \sin \theta & e^{i \chi} \cos \theta
\end{array}\right)\left(\begin{array}{cc}
u_{I}^{T} & 0 \\
0 & u_{I}^{\dagger}
\end{array}\right), \\
\sin \theta=\Gamma\left\{\left[1-e^{2 i \phi_{A}}(1-\Gamma)\right]\left[1-e^{-2 i \phi_{A}}(1-\Gamma)\right]\right\}^{-1 / 2} \\
e^{2 i \chi}=\left(1-\Gamma-e^{2 i \phi_{A}}\right)\left[1-e^{2 i \phi_{A}}(1-\Gamma)\right]^{-1}
\end{gathered}
$$

The left matrix $r_{L}$ is given by Eq. (15) and describes the reflection from the disordered wire. Taking advantage of the polar decomposition we can write

$$
r_{L}=\left(\begin{array}{cc}
u_{0} & 0 \\
0 & u_{0}^{*}
\end{array}\right)\left(\begin{array}{cc}
e^{i \phi} & 0 \\
0 & e^{i \phi}
\end{array}\right)\left(\begin{array}{cc}
u_{0}^{T} & 0 \\
0 & u_{0}^{\dagger}
\end{array}\right),
$$

where $u_{0}$ is a random unitary matrix and $\phi$ is the diagonal matrix of the eigenphases. We see that all information contained in $u_{I}$ disappears statistically from the eigenvalues of $r_{L} r_{R}$ because the product $u_{I}^{T} u_{0}$ can be regarded again as a random unitary matrix. Thus the disorder-averaged LDOS depends only on the transmission eigenvalues $\Gamma_{j}$ of the tunnel barrier. Below we calculate the mean LDOS for a singlechannel wire and for a multichannel wire provided the tunneling probabilities are the same for all channels, i.e., $\Gamma_{j}$ $=\Gamma$.

\section{B. Single channel wire}

We start with the calculation of the mean LDOS for $\varepsilon$ $<\Delta$ in the case of the single-channel wire $N=1$. For $\varepsilon<\Delta$ the phases $\chi$ and $\theta$ defined in Eq. (35) are real and both $r_{L}$ and $r_{R}$ are unitary $2 \times 2$ matrices. We denote $u_{I}^{T} u_{0}$ $=\exp (i \psi)$, where $\psi$ is a random phase distributed uniformly in the interval $(0,2 \pi)$. We insert the reflection matrices from Eqs. (35a),(36) directly to Eq. (13). The matrix $\left(1-r_{L} r_{R}\right)$ can be easily inverted. Taking the real part we notice that the zeroes of $\operatorname{det}\left(1-r_{L} r_{R}\right)$ define the exact positions of the quasiparticle bound states for $\varepsilon<\Delta$. The result reads

$$
n(x, \varepsilon)=\pi \sin (\phi+\chi) \delta[\cos \theta \cos \psi-\cos (\phi+\chi)],
$$

where the argument of the Dirac $\delta$ function corresponds to the quantization condition for the bound states. The mean LDOS is given by the average over the phase $\phi$ with the probability density $\rho_{\varepsilon}(\phi)$ of Eq. (19), and over the uniformly distributed phase $\psi$. The integration over $\psi$ is readily done with the result 


$$
\bar{n}(x, \varepsilon)=\int_{\theta-\chi}^{\pi-\theta-\chi} d \phi \rho_{\varepsilon}(\phi) \frac{\sin (\phi+\chi)}{\sqrt{\cos ^{2} \theta-\cos ^{2}(\phi+\chi)}} .
$$

In the limit $\Gamma \rightarrow 1$ of the vanishing tunnel barrier one observes that $\chi \rightarrow \pi / 2-\phi_{A}$ and $\theta \rightarrow \pi / 2$, so that the area of the integration in Eq. (37) shrinks to the small vicinity of $\phi$ $=\phi_{A}$ and the function $\rho_{\varepsilon}(\phi)$ can be substituted by its value in this point. The integral approaches $\pi$ and we recover the result of Eq. (17) for the ideally transmitting interface.

In the opposite extreme of a high tunnel barrier $(\Gamma \rightarrow 0)$ both $\theta$ and $\chi$ go to zero, so that the integration area is not restricted and the value of the integral tends to unity because of the normalization condition for the probability density $\rho_{\varepsilon}(\phi)$. In the limit $\varepsilon \ll \Delta$ we can set $\chi=0$ and reduce Eq. (38) to the following form:

$$
\bar{n}(x, \varepsilon)=\operatorname{Re} \int_{0}^{\infty} d t e^{-t}\left[1-\frac{\sin ^{2} \theta}{\left(\varepsilon \tau_{s}\right)^{2}} t\left(t-2 i \varepsilon \tau_{s}\right)\right]^{-1 / 2},
$$

where $\sin \theta=\Gamma /(2-\Gamma)$, according to Eq. (35b). From Eq. (39) we find that

$$
\bar{n}(x, \varepsilon)=\pi \varepsilon \tau_{s} \frac{2-\Gamma}{2 \Gamma}, \quad \varepsilon \tau_{s} \ll \frac{\Gamma}{2-\Gamma},
$$

which coincides for $\Gamma=1$ with the result of Eq. (30) for $N$ $=1$. In the dirty limit $\Delta \ll \tau_{s}^{-1}$ and for a high tunnel barrier $\Gamma \ll 1$ the result of Eq. (39) is applicable almost up to the value of $\varepsilon=\Delta$. It describes the formation of the pseudo-gap near the energy $\tau_{s}^{-1} \Gamma$ due to the normal reflection from the barrier.

The exact expression (38) additionally accounts for the peak at $\varepsilon \simeq \Delta$. This expression can be further generalized for energies higher than $\Delta$ by means of the analytical continuation $\varepsilon=i \omega$, with the result

$$
\begin{aligned}
& \bar{n}(x, \varepsilon)=-\operatorname{Re} \int_{0}^{\infty} d \sigma \rho_{1}(\sigma) \frac{\sinh Q(\sigma)}{\sqrt{\sinh ^{2} Q(\sigma)+\sin ^{2} \theta}}, \\
& Q(\sigma)=\frac{1}{2} \ln \frac{\alpha^{2}(\omega)+1-\Gamma}{1+\alpha^{2}(\omega)(1-\Gamma)}+\frac{1}{2} \ln \frac{\sigma}{\sigma+4 \omega \tau_{s}},
\end{aligned}
$$

where the function $\rho_{1}(\sigma)=(1 / 2) \exp (-\sigma / 2)$ is the probability density (24) for a single-channel wire, the function $\alpha(\omega)$ is defined in Eq. (21), and the continuation to the real energies $\omega \rightarrow-i \varepsilon+0^{+}$is performed (see Fig. 4).

\section{Multichannel wire}

The disorder-averaged LDOS for $N \gg 1$ can be found straightforwardly for the case of equivalent tunnelling probabilities $\Gamma_{j}=\Gamma$. Then the diagonal matrices $\theta$ and $\chi$ in Eqs. $(35 b),(35 c)$ can be regarded as scalars. It is convenient to make use of the analytical continuation $\varepsilon=i \omega$ and define

$$
\alpha(\omega)=i e^{i \phi_{A}}, \quad R=e^{2 i \phi},
$$

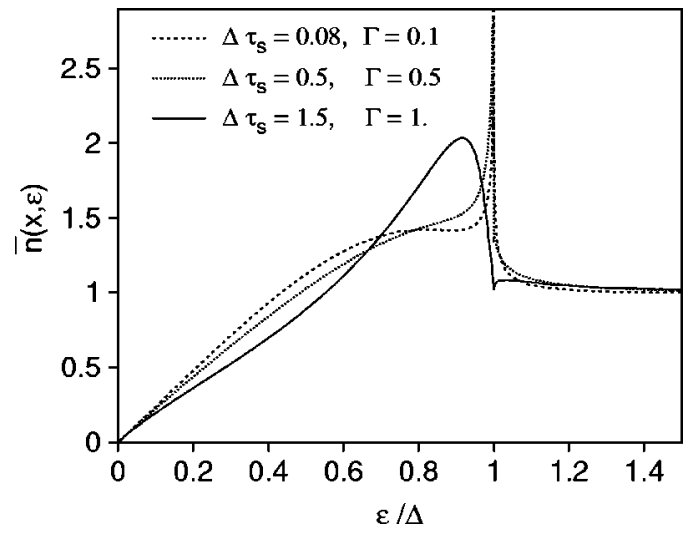

FIG. 4. The mean LDOS (41) in a single-channel disordered wire at ballistic distance from an NS interface of finite transparency. The parameters $\Delta \tau_{s}$ and $\Gamma=0.5$ are chosen to fix the combination $\Delta \tau_{s}(2-\Gamma) / \Gamma \approx 1.5$. The curves are close to each other for $\varepsilon \ll \Delta$ where Eq. (39) is applicable.

$$
p=e^{2 i \chi}=\frac{\alpha^{2}(\omega)+1-\Gamma}{1+\alpha^{2}(\omega)(1-\Gamma)},
$$

where $R=\operatorname{diag}\left(R_{1}, \ldots, R_{N}\right)$ is the diagonal matrix of reflection probabilities for the disordered wire with a fictitious absorption $\omega$. In the parametrization (22) the joint probability density of $R_{j}$ is related to the orthogonal Laguerre ensemble (23). Note that the quantities $p, R_{j}$, and $\alpha(\omega)$ take real values in the interval $(0,1)$ when $\omega$ is real.

The basic expression (14) for the mean LDOS is manifestly invariant under an arbitrary unitary rotation of the matrix product $r_{L} r_{R}$. From Eqs. (35a),(36) we obtain

$$
\begin{aligned}
U_{0}^{\dagger} r_{L} r_{R} U_{0} & =\left(\begin{array}{cc}
\sqrt{p R} & 0 \\
0 & \sqrt{p R}
\end{array}\right)\left(\begin{array}{ccc}
\cos \theta & U & -i \sin \theta \\
-i \sin \theta & \cos \theta & U^{\dagger}
\end{array}\right), \\
U & =u_{0}^{T} u_{I} u_{I}^{T} u_{0}, \quad U_{0}=\operatorname{diag}\left(u_{0}, u_{0}^{*}\right),
\end{aligned}
$$

where we take advantage of the quantities defined in Eq. (42). The matrix $u_{0}$ is a random unitary matrix which is uniformly distributed in the unitary group (provided the weak disorder $k_{F} l \gg 1$ ). Hence by construction (43b), $U$ is the unitary symmetric random matrix. We substitute Eq. (43a) into Eq. (14) to express the mean LDOS as

$$
\bar{n}(x, \varepsilon)=\frac{1}{N} \operatorname{Re} \operatorname{Tr}\left\langle\frac{1-p R}{1+p R}\langle F(\cos \theta)\rangle_{U}\right\rangle_{R},
$$

where

$$
\begin{gathered}
F(z)=\frac{1}{1-z\left(\sqrt{C_{1}} U \sqrt{C_{2}}+\sqrt{C_{2}} U^{\dagger} \sqrt{C_{1}}\right)}, \\
C_{1}=\frac{p R}{1+p R}, \quad C_{2}=\frac{1}{1+p R} .
\end{gathered}
$$

The average over disorder in Eq. (44) is decoupled into two independent steps: the average $\langle\cdots\rangle_{U}$ over the group 
spanned by the unitary symmetric matrices and the average $\langle\cdots\rangle_{R}$ over the orthogonal Laguerre ensemble of the reflection eigenvalues $R_{j}$.

In the case of the finite number of channels the calculation of average over the unitary matrices $U$ is technically difficult and cannot be done analytically. However, for the diffusive wire, $N \gg 1$, the calculation can be done by means of the diagrammatic technique developed in Ref. 37.

Let us briefly quote the basic substitution rules of the diagrammatic technique

$$
\begin{array}{ll}
U_{i j}=\bullet-\cdots & U_{i j}^{*}=\bullet-\cdots-\mathrm{o}, \\
C_{i j}=\stackrel{\mathrm{c}}{\longrightarrow}, & \delta_{i j}=\longrightarrow .
\end{array}
$$

Here the matrix element $U_{i j}$ is represented by the black and white dot connected by the dashed line. The black dot stays for the first index $i$ and the white dot for the second index $j$. The conjugated matrix $U^{*}$ is marked by an asterisk. The other matrices are denoted by thick solid arrows. The summation over a matrix index in a dot is indicated by the attachment of a solid line. The average over the unitary symmetric matrices is symbolically performed by pairing in all possible ways all black and white dots belonging to $U$ to all black and white dots belonging to $U^{*}$. This pairing is denoted by the thin solid line, which corresponds to the Kronecker symbol. The result of the averaging is found by inspection of the closed circuits in the diagram which consist of alternating thick and thin solid lines ( $T$ circles). Each diagram is weighted by a factor, which is obtained by inspection of the closed circuits of alternating thin solid and dashed lines ( $U$ circles).

We expand the matrix $F(z)$ (45) into a geometric series and keep only the terms with equal number of $U$ and $U^{\dagger}$ matrices. In the large- $N$ limit we have to take into account the diagrams with the largest number of $T$ circles. ${ }^{37}$ This amounts to the summation of the "rainbow" diagrams, or diffusion ladders, depicted symbolically in Fig. 5. The corresponding Dyson equation is

$$
\begin{gathered}
\langle F\rangle_{U}=\hat{1}+z \Sigma_{1} C_{1}\langle F\rangle_{U}+z \Sigma_{2} C_{2}\langle F\rangle_{U}, \\
\Sigma_{1}=\sum_{n=1}^{\infty} W_{n} z^{n-1}\left[\operatorname{Tr} C_{2}\langle F\rangle_{U}\right]^{n}\left[\operatorname{Tr} C_{1}\langle F\rangle_{U}\right]^{n-1}, \\
\Sigma_{2}=\sum_{n=1}^{\infty} W_{n} z^{n-1}\left[\operatorname{Tr} C_{1}\langle F\rangle_{U}\right]^{n}\left[\operatorname{Tr} C_{2}\langle F\rangle_{U}\right]^{n-1},
\end{gathered}
$$

where the weight factors

$$
W_{n}=N^{1-2 n}(-1)^{n-1} \frac{(2 n-2) !}{n !(n-1) !}+\mathcal{O}\left(N^{-2 n}\right)
$$

have been found in Ref. 37. Taking the coefficients $W_{n}$ to the leading order in $N$ we define the generating function

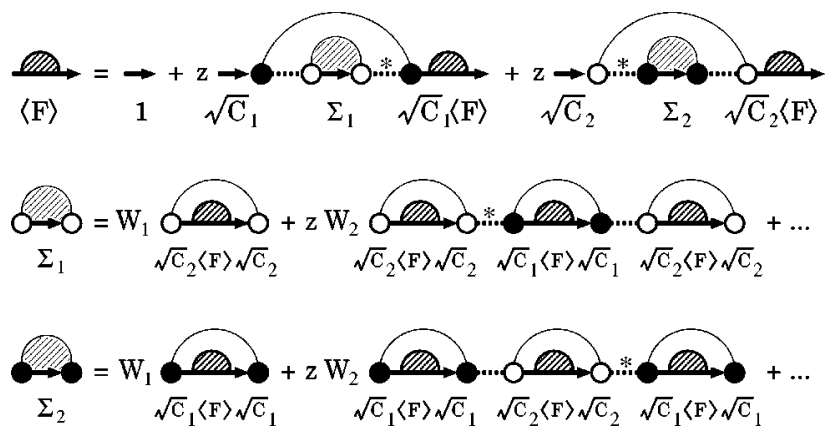

FIG. 5. Diagrammatic representation of the Dyson equation (47) for $\langle F(z)\rangle_{U}$.

$$
h(s)=\sum_{n=1}^{\infty} W_{n} s^{n-1}=\frac{1}{2 s}\left(\sqrt{N^{2}+4 s}-N\right),
$$

which may be used to reduce Eq. (47) to

$$
\begin{gathered}
\langle F(z)\rangle_{U}=\hat{1}+z^{2} h\left(z^{2} s_{1} s_{2}\right)\left(s_{1} C_{1}+s_{2} C_{2}\right)\langle F(z)\rangle_{U}, \\
s_{1,2}=\operatorname{Tr} C_{1,2}\langle F(z)\rangle_{U} .
\end{gathered}
$$

The matrix $\langle F(z)\rangle_{U}$ has to be eliminated from the Dyson equations (50). After that it is very convenient to transform to the new scalar variables

$$
X=\frac{s_{2}-s_{1}}{N}, \quad Y=\frac{s_{2}+s_{1}}{N},
$$

which obey the equations

$$
\begin{gathered}
\frac{X+1}{2}=\frac{1}{N} \operatorname{Tr} \frac{1}{1+p R f(X, Y)}, \\
Y^{2} \sin ^{2} \theta+X^{2} \cos ^{2} \theta=1,
\end{gathered}
$$

with

$$
f(X, Y)=\frac{(1-X)(Y+X)}{(1+X)(Y-X)},
$$

where we have substituted $z=\cos \theta$ and the matrices $C_{1}, C_{2}$ from Eq. (45b).

In terms of the variables $X$ and $Y$ the mean LDOS (44) is simplified to

$$
\bar{n}(x, \varepsilon)=\left.\operatorname{Re} \bar{X}(\omega)\right|_{\omega \rightarrow-i \varepsilon+0^{+}},
$$

where the bar stands for the average over the ensemble of the reflection probabilities $\bar{X} \equiv\langle X\rangle_{R}$.

Let us first consider the case of equal reflection probabilities $R_{j}=R$. The matrix $U$ in Eq. (45) commutes with $C_{1}$ and $C_{2}$ and can be diagonalized, hence the problem becomes equivalent to that of a single channel wire. The solution of the self-consistent equations Eq. (52) is given by

$$
X=-\frac{\sinh Q}{\sqrt{\sinh ^{2} Q+\sin ^{2} \theta}}, \quad Q=\frac{1}{2} \ln p R,
$$


which coincides with the result of the exact integration over $U$. This proves that the set of diagrams which we took into account in Eqs. (47) is sufficiently complete. The substitution of Eq. (55) in Eq. (54) and the additional average over the reflection probability of a single channel wire yields the mean LDOS of Eq. (41).

In the multichannel (diffusive) limit $N \gg 1$ the reflection probabilities $R_{j}$ are, in fact, not equal. Moreover they effectively repel each other according to Eqs. (22),(23). In this case Eq. (52) can no longer be solved in closed form. In other words, the averages over the random matrix $U$ and over the reflection eigenvalues $R_{j}$ cannot be performed separately.

In order to proceed one has to take advantage of the selfaveraging property of the variables $X$ and $Y$ in the limit $N$ $\gg 1$. Indeed both variables are defined via the traces $s_{1,2}$ and can be thought as the arithmetic means of $N$ fluctuating quantities. From a physical point of view the variable $X$ is proportional to the one-point Green function, therefore it is self-averaging in a diffusive metal.

Thus we can construct the self-consistent equation for $\bar{X}$ by taking the average over $R$ on both sides of Eq. (52a). We assume a fixed value of $f[X, Y(X)]=\widetilde{f}(\bar{X})$ on the right side, neglecting the fluctuations of $X$. Taking advantage of the square-root approximation (26) of the density $\rho(\sigma)$ we obtain

$$
\frac{\bar{X}+1}{2}=\frac{1}{2 \pi} \int_{0}^{4} d \zeta \sqrt{\frac{4}{\zeta}-1} \frac{2 \omega \tau_{s}+\zeta}{2 \omega \tau_{s}+[1+p \widetilde{f}(\bar{X})] \zeta} .
$$

The integral on the right-hand side can be carried out explicitly giving rise to the equation

$$
\begin{aligned}
& \frac{\left[\alpha^{2}(\omega)+1-\Gamma\right][Y(\bar{X})+\bar{X}]}{\left[1+\alpha^{2}(\omega)(1-\Gamma)\right][Y(\bar{X})-\bar{X}]} \\
& \quad=1+\frac{2}{1+\bar{X}}\left(\omega \tau_{s}-\sqrt{\omega \tau_{s}} \sqrt{1+\bar{X}+\omega \tau_{s}}\right)
\end{aligned}
$$

which is an algebraic equation for $\bar{X}$. It can be analytically continued to real energies $\omega=-i \varepsilon+0^{+}$and solved numerically by iteration. The disorder-averaged LDOS is determined, then, from Eq. (54). Equation (57) is obtained in the quasiclassical limit of a large number of channels. This result does not change if we neglect that $U$ is symmetric or take the unitary Laguerre ensemble in Eq. (23) instead of the orthogonal one.

The weak-localization correction (which we simply define as $1 / N$ correction) can, in principle, be determined within the present approach. It has three different sources. First of all an additional class of diagrams, namely the Cooperon-like diagrams, have to be taken into account in the Dyson equation (47). Secondly the term of subleading order in the large- $N$ expansion of the weight factors $W_{n}$ has to be included. Finally the correction of order $\mathcal{O}\left(N^{-1}\right)$ to the limiting form (26) of the probability density $\rho(\sigma)$ has to be considered. The calculation of the weak localization correction to the LDOS is, however, beyond the scope of this paper.
In some limiting cases Eq. (57) allows for a transparent analytical solution. In the absence of the tunnel barrier $\Gamma$ $=1$ we obtain

$$
\bar{X}=1-\left.2 \frac{\alpha^{2}}{1+\alpha^{2}}(1+\Omega-\sqrt{\Omega} \sqrt{2+\Omega})\right|_{\Omega \rightarrow \omega \tau_{s} /\left(1+\alpha^{2}\right)},
$$

which coincides upon the substitution in Eq. (54) with the result of Eq. (25) in the large- $N$ limit. In the limit $\Delta \tau_{s}$ $\ll \Gamma^{2}$, Eq. (57) leads to the BCS result for the local density, Eq. (27).

For small energies, $\omega \ll \Delta$, we can put $\alpha(\omega)=1$ and obtain

$$
\bar{X}=\frac{\sqrt{\omega \tau_{s}} \sqrt{4 \sin ^{2} \theta+\omega \tau_{s}}-\omega \tau_{s}}{2 \sin ^{2} \theta} .
$$

The mean LDOS (54) for $\varepsilon \ll \Delta$ is then given by

$$
\bar{n}(x, \varepsilon)=\operatorname{Re} \sqrt{-i \frac{\varepsilon \tau_{s}}{\sin ^{2} \theta}} \sqrt{1-i \frac{\varepsilon \tau_{s}}{4 \sin ^{2} \theta}},
$$

with $\sin \theta=\Gamma /(2-\Gamma)$. This result describes the scaling $\varepsilon_{g}$ $\sim \tau_{s}^{-1} \Gamma^{2}(2-\Gamma)^{-2}$ of the size of the pseudogap $\varepsilon_{g}$ with the transparency of the tunnel barrier $\Gamma$, which is illustrated in Fig. 7. We observe that in the limit $\Gamma^{2} \ll \Delta \tau_{s} \ll 1$ two different types of bound states contribute to the LDOS at energies below $\Delta$. One group of the bound states is responsible for the monotonous increase of the LDOS to its bulk value at the scale $\tau_{s}^{-1} \Gamma^{2}$ while another group gives rise to the formation of the peak near $\varepsilon=\Delta$.

\section{USADEL EQUATION}

The aim of this section is to compare our results in the limit $N \gg 1$ to the results of the conventional quasiclassical theory based on the Usadel equation. It is important to remember that the Usadel description is justified only in the dirty limit $\Delta \tau_{s} \ll 1$, while it is not restricted to the clean superconducting material as is the case with our calculation. In the quasiclassical context the superconductor as well as the normal metal are characterized by their diffusion constants $D_{s}, D_{n}$ and normal-state resistivities $\rho_{s}, \rho_{n}$, which are combined into the mismatch parameter

$$
\gamma=\frac{\rho_{s} \xi_{s}}{\rho_{n} \xi_{n}},
$$

where $\xi_{n, s}=\sqrt{D_{n, s} / \Delta}$ are the diffusive coherence lengths. Hence, the comparison has to be done in the limit $\gamma \ll 1$, where the "rigid" boundary condition is valid.

In the case of the perfectly transparent $N S$ boundary and vanishing mismatch parameter the LDOS at the interface found from the Usadel equation ${ }^{13,15}$ is simply given by the standard BCS formula and, therefore, coincides with our expression (27) in the dirty limit $\Delta \tau_{s} \ll 1$. Thus, there is not too much to compare for the case of transparent boundary. However, if the NS interface is not perfectly transparent $(\Gamma$ 

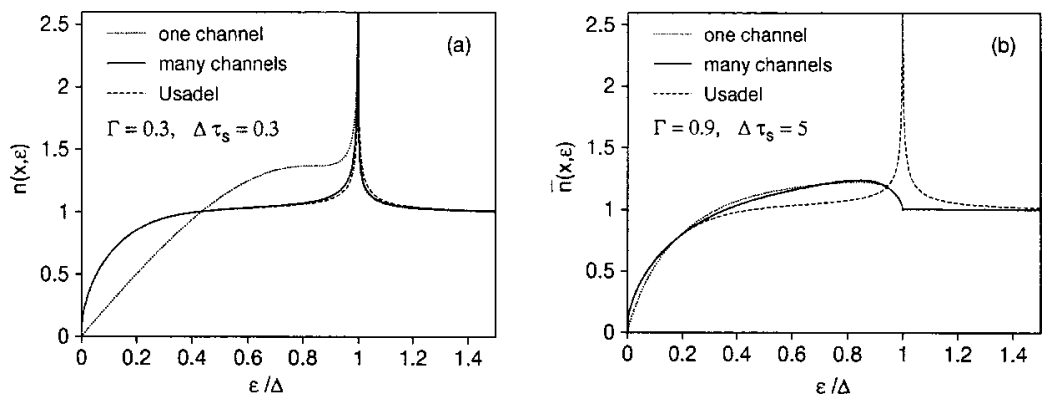

FIG. 6. The mean LDOS in a normal-metal wire in the vicinity of an $N S$ interface of finite transparency. The dotted curve is found from Eq. (41) for the single-channel wire $N=1$. The solid curve is calculated from Eqs. (54),(57) for the diffusive wire $N \gg 1$. The dashed curve represents the result of the Usadel equation, Eqs. (65),(69), calculated for the corresponding value of the parameter $\gamma_{B}^{2}=\Delta \tau_{s}(2$ $-\Gamma)^{2} /(2 \Gamma)^{2}$. The figures show the energy dependence of the mean LDOS for the dirty $\Delta \tau_{s}=0.3$ and the clean $\Delta \tau_{s}=5$ regime.

$<1$ ), even the limit of small mismatch parameter $\gamma$ is not completely trivial. Let us now discuss the Usadel equation for this case in somewhat more detail following the calculation of Ref. 14.

The transparency of the interface enters the theory through the parameter

$$
\gamma_{B}=\frac{R_{B}}{\rho_{n} \xi_{n}}
$$

where $R_{B}$ is the product of the barrier resistance and its area. The Usadel equation in the normal metal $(x<0)$ takes the form

$$
\frac{D_{n}}{2} \Theta_{n}^{\prime \prime}(x)-\omega \sin \Theta_{n}(x)=0
$$

where $\omega=-i \varepsilon+0^{+}$is the imaginary energy, while in the superconductor $(x>0)$ the equation reads

$$
\frac{D_{s}}{2} \Theta_{s}^{\prime \prime}(x)-\omega \sin \Theta_{s}(x)+\Delta(x) \cos \Theta_{s}(x)=0,
$$

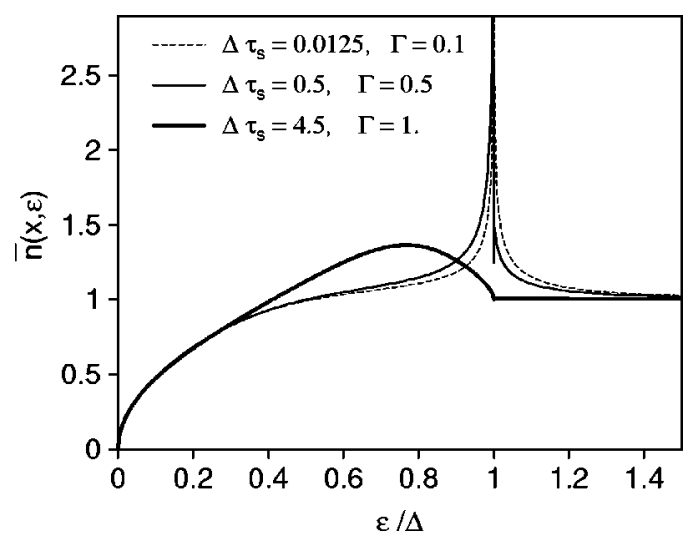

FIG. 7. The mean LDOS in vicinity of an $N S$ interface of finite transparency calculated from Eqs. (54),(57). The parameters $\Delta \tau_{s}$ and $\Gamma=0.1$ are chosen to fix the combination $\gamma_{B}^{2}=\Delta \tau_{s}(2$ $-\Gamma)^{2} /(2 \Gamma)^{2} \approx 1.06$. The curves coincide for $\varepsilon \ll \Delta$, where Eqs. (60),(71) are applicable. The result of the Usadel equation, Eqs. (65),(69), is indistinguishable from the dashed line. where $\Delta(x)$ is the gap function. (For a sake of simplicity we restrict ourselves to zero temperature.) The functions $G(x, x)=\cos \Theta_{n, s}(x)$ and $F(x, x)=\sin \Theta_{n, s}(x)$ parametrize normal and anomalous quasiclassical Green functions in energy representation, averaged over angle and disorder. The LDOS near the interface is given by

$$
\bar{n}(0, \varepsilon)=\operatorname{Re} \cos \Theta_{n}(0) .
$$

Far away from the NS interface the Green functions aquire their bulk values

$$
\cos \Theta_{n}(-\infty)=1, \quad \cos \Theta_{s}(\infty)=\frac{\omega}{\sqrt{\Delta^{2}+\omega^{2}}} .
$$

The finite transparency of the interface comes into play in the appropriate matching conditions at $x=0^{12}$

$$
\begin{gathered}
\gamma_{B} \xi_{n} \Theta_{n}^{\prime}(0)=\sin \left[\Theta_{s}(0)-\Theta_{n}(0)\right], \\
\gamma \xi_{n} \Theta_{n}^{\prime}(0)=\xi_{s} \Theta_{s}^{\prime}(0) .
\end{gathered}
$$

Once the superconductor is sufficiently clean the first term in Eq. (64) can be disregarded, hence $\Theta_{s}(x)=\Theta_{s}(\infty)$ and $\Delta(x)=\Delta$ for $x>0$. This justifies the "rigid" boundary conditions, which are used throughout the article.

The first integral of Eq. (63) is readily found

$$
\frac{D_{n}}{4}\left[\Theta_{n}^{\prime}(x)\right]^{2}+\omega \cos \Theta_{n}(x)=\text { const }
$$

where the constant is determined from the condition at $x$ $=-\infty$ and equals $\omega$. With the help of Eq. (67a) one obtains $^{38}$

$$
\frac{\sin ^{2}\left[\Theta_{n}(0)-\Theta_{s}(0)\right]}{4 \gamma_{B}^{2}}+\frac{\omega}{\Delta}\left[\cos \Theta_{n}(0)-1\right]=0,
$$

where $\Theta_{s}(0)$ is substituted by $\Theta_{s}(\infty)$ due to the "rigid" boundary condition. In the limit $\omega \ll \Delta$ the equation is simplified to

$$
\left(\cos \Theta_{n}(0)-\frac{\omega}{\Delta}\right)^{2}=\frac{4 \gamma_{B}^{2} \omega}{\Delta}\left[1-\cos \Theta_{n}(0)\right] .
$$

Its solution gives rise to the LDOS for $\varepsilon \ll \Delta$ 


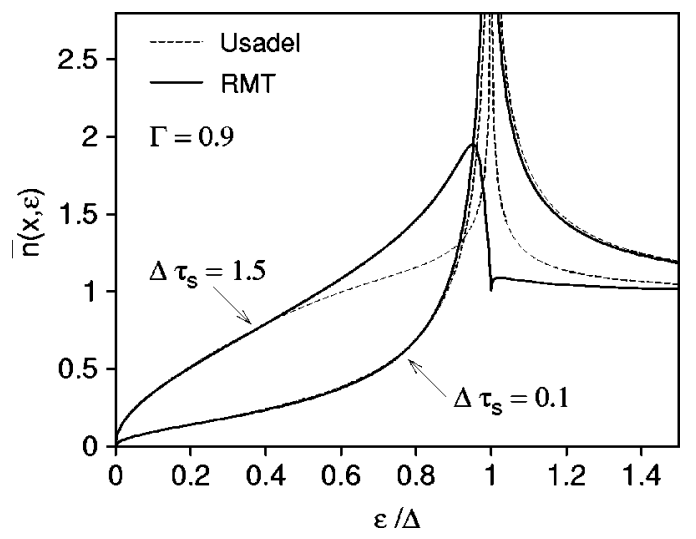

FIG. 8. The mean LDOS from the random matrix theory (solid lines), Eqs. (54),(57), is compared to that from the Usadel theory (dashed lines), Eqs. (65),(69), for the corresponding value of the parameter $\gamma_{B}^{2}=\Delta \tau_{s}\left(2-\Gamma^{2}\right) /(2 \Gamma)^{2}$. The curves always coincide for small energies $\varepsilon \ll \Delta$. The perfect agreement in the entire energy range is found in the dirty limit $\Delta \tau_{s} \rightarrow 0$, where the Usadel equation is justified.

$$
\bar{n}(0, \varepsilon)=\operatorname{Re} \sqrt{-i \frac{4 \varepsilon \gamma_{B}^{2}}{\Delta}} \sqrt{1-i \frac{\varepsilon \gamma_{B}^{2}}{\Delta}},
$$

which is manifestly equivalent to Eq. (60) and establishes the following relation between the parameters:

$$
\gamma_{B}^{2}=\Delta \tau_{s} \frac{(2-\Gamma)^{2}}{4 \Gamma^{2}} .
$$

This relation also follows directly from the definition of $\gamma_{B}$, up to a numerical factor, since one can effectively substitute $R_{B}=\left(h^{2} / e\right)(2-\Gamma) / 2 \Gamma, \rho_{n}=\left(h^{2} / e\right) l^{-1}$, and $D_{n}=l^{2} / \tau_{s}$.

We conclude that the LDOS obtained from the Usadel equation always coincides with that found from Eq. (57) for small energies $\varepsilon \ll \Delta$. We also demonstrate numerically in Figs. 6,7 , and 8 that our result for $N \gg 1$ is perfectly consistent with the Usadel theory in the dirty limit $\Delta \tau_{s} \ll 1$, where the latter is justified.

One should note, however, that the agreement with the quasiclassical theory becomes better with the increasing barrier height. Indeed, in the perfectly transparent interface $\Gamma$ $=1$, the agreement is reached only in the extremely dirty limit $\Delta \tau_{s} \rightarrow 0$, while for smaller values of $\Gamma$ the dirty-limit condition is less restrictive [see Fig. 6(a)].

\section{CONCLUSION}

In conclusion, we computed the mean LDOS in a normalmetal disordered wire in the immediate vicinity of an $N S$ interface at zero temperature and zero magnetic field. Our calculation is based on the scattering approach and takes into account the spatial phase coherence in the normal metal.

We derived the general formula (10), which expresses the one-point Green function in terms of the reflection matrices. The formula can be applied in order to calculate the LDOS (and its distribution) in the wire at arbitrary distance to the $N S$ interface. In this paper we only considered the mean LDOS at the ballistic distance to the interface so that it does not acquire a spatial dependence.

We obtained the relation (1) between the disorderaveraged LDOS near the ideal NS interface and the probability density of the eigenphases of the matrix correlator $r_{0}(\varepsilon) r_{0}(-\varepsilon)^{\dagger}$, where $r_{0}(\varepsilon)$ is the reflection matrix for the semi-infinite normal-metal wire.

We also study in detail the case of the normalsuperconductor tunnel junction and derive the self-consistent equation (57) that determines the LDOS in the diffusive normal metal. In the dirty limit our expression coincides with the LDOS found by Golubov and Kupriyanov ${ }^{14}$ from the Usadel equation.

The quasiclassical analysis of the Green function at the $N S$ interface of finite transparency has been performed by many authors $12,39,14,40,41$ in connection with the boundary conditions of semiclassical superconductivity. However, to our best knowledge no counterpart to Eq. (57) exists in the literature.

In the case of an ideal NS interface the LDOS is found to be almost independent of the number of channels in a wire, except for very small energies, hence its insensitivity to phase-coherence effects. This persists to the case of finite transparency provided the clean limit condition $\Delta \tau_{s} \gg 1$. In the dirty limit $\Delta \tau_{s} \ll 1$ and small transparency $\Gamma \ll 1 / N$ the situation is different and the phase-coherent effects play a role.

The effect of Anderson localization is seen in the linear increase of the LDOS, $\bar{n}=(\pi / 4)(N+1) \varepsilon \tau_{s}(2-\Gamma) / \Gamma$, for energies lower than $\varepsilon_{c}=1 / N^{2} \tau_{s}$. In the diffusive metal, $N$ $\rightarrow \infty$, the LDOS increases as the square root of energy $\bar{n}$ $=\operatorname{Re} \sqrt{-i \varepsilon \tau_{s}}(2-\Gamma) / \Gamma$. The form of the crossover in energy dependence of the LDOS from linear to square-root behavior is given by Eq. (25) for weak disorder and perfect $N S$ interface.

\section{ACKNOWLEDGMENTS}

We thank C. W. J. Beenakker, P. W. Brouwer, and R. Narayanan for helpful discussions. We are especially grateful to Alexander Golubov for bringing the results of Ref. 14 to our attention.
${ }^{1}$ L. N. Cooper, Phys. Rev. Lett. 6, 689 (1961).

${ }^{2}$ G. Deutscher and P. G. de Gennes, in Superconductivity, edited by

R. D. Parks (Dekker, New York, 1969), Vol. 2, p. 1005.

${ }^{3}$ Mesoscopic Electron Transport, edited by L. P. Kouwenhoven, G.
Schön, and L. L. Sohn, Vol. 345 of NATO Advanced Studies Institute, Series E: Applied Sciences (Kluwer Academic Publishers, Dordrecht, 1997).

${ }^{4}$ A. F. Andreev, Zh. Éksp. Teor. Fiz. 46, 1823 (1964) [Sov. Phys. 
JETP 19, 1228 (1964)].

${ }^{5}$ R. Fazio and C. Lucheroni, Europhys. Lett. 45, 707 (1999); I. Baladié and A. Buzdin, Phys. Rev. B 64, 224514 (2001); K. Halterman and O. T. Valls, ibid. 65, 014509 (2001); F. S. Bergeret, A. F. Volkov, and K. B. Efetov, ibid. 65, 134505 (2002).

${ }^{6}$ M. A. Sillanpää, T. T. Heikkilä, R. K. Lindell, and P. J. Hakonen, Europhys. Lett. 56, 590 (2001).

${ }^{7}$ S. H. Tessmer, D. J. Van Harlingen, and J. W. Lyding, Phys. Rev. Lett. 70, 3135 (1993); S. H. Tessmer, M. B. Tarlie, D. J. Van Harlingen, D. L. Maslov, and P. M. Goldbart, ibid. 77, 924 (1996).

${ }^{8}$ S. Guëron, H. Pothier, N. O. Birge, D. Esteve, and M. H. Devoret, Phys. Rev. Lett. 77, 3025 (1996).

${ }^{9}$ Y. Levi, O. Millo, N. D. Rizzo, D. E. Prober, and L. R. Motowidlo, Phys. Rev. B 58, 15128 (1998).

${ }^{10}$ N. Moussy, H. Courtois, and B. Pannetier, Europhys. Lett. 55, 861 (2001).

${ }^{11}$ M. Vinet, C. Chapelier, and F. Lefloch, Phys. Rev. B 63, 165420 (2001).

${ }^{12}$ M. Yu. Kupriyanov and V. F. Lukichev, Zh. Éksp. Teor. Fiz. 94, 139 (1988) [Sov. Phys. JETP 67, 1163 (1988)].

${ }^{13}$ A. A. Golubov and M. Yu. Kupriyanov, J. Low Temp. Phys. 70, 83 (1988)

${ }^{14}$ A. A. Golubov and M. Yu. Kupriyanov, Physica C 259, 27 (1996).

${ }^{15}$ W. Belzig, C. Bruder, and G. Schön, Phys. Rev. B 54, 9443 (1996).

${ }^{16}$ S. Pilgram, W. Belzig, and C. Bruder, Physica B 280, 442 (2000); Phys. Rev. B 62, 12462 (2000).

${ }^{17}$ Ya. V. Fominov and M. V. Feigelman, Phys. Rev. B 63, 094518 (2001)

${ }^{18}$ K. D. Usadel, Phys. Rev. Lett. 25, 507 (1970).

${ }^{19}$ G. Eilenberger, Z. Phys. 214, 195 (1968).

${ }^{20}$ K. K. Likharev, Rev. Mod. Phys. 51, 101 (1979).

${ }^{21}$ M. Titov and C. W. J. Beenakker, Phys. Rev. Lett. 85, 3388 (2000).
${ }^{22}$ M. Schechter, Y. Imry, and Y. Levinson, Phys. Rev. B 64, 224513 (2001).

${ }^{23}$ C. W. J. Beenakker, Rev. Mod. Phys. 69, 731 (1997).

${ }^{24}$ O. N. Dorokhov, Pis'ma Zh. Éksp. Teor. Fiz. 36, 259 (1982) [JETP Lett. 36, 318 (1982)].

${ }^{25}$ P. A. Mello, P. Pereyra, and N. Kumar, Ann. Phys. (N.Y.) 181, 290 (1988).

${ }^{26}$ H. Schomerus, M. Titov, P. W. Brouwer, and C. W. J. Beenakker, Phys. Rev. B 65, 121101(R) (2002).

${ }^{27}$ D. S. Fisher and P. A. Lee, Phys. Rev. B 23, 6851 (1981).

${ }^{28}$ V. Gasparian, T. Christen, and M. Büttiker, Phys. Rev. A 54, 4022 (1996).

${ }^{29}$ M. Titov, N. A. Mortensen, H. Schomerus, and C. W. J. Beenakker, Phys. Rev. B 64, 134206 (2001).

${ }^{30}$ V. L. Berezinskii and L. P. Gor'kov, Sov. Phys. JETP 50, 1209 (1979).

${ }^{31}$ B. White, P. Sheng, Z. Q. Zhang, and G. Papanicolaou, Phys. Rev. Lett. 59, 1918 (1987).

${ }^{32}$ M. Titov, P. W. Brouwer, A. Furusaki, and C. Mudry, Phys. Rev. B 63, 235318 (2001).

${ }^{33}$ M. L. Mehta, Random Matrices (Academic, New York, 1991).

${ }^{34}$ A. Altland and M. R. Zirnbauer, Phys. Rev. B 55, 1142 (1997).

${ }^{35}$ K. Slevin and T. Nagao, Phys. Rev. B 50, 2380 (1994).

${ }^{36}$ T. Nagao and K. Slevin, J. Math. Phys. 34, 2317 (1993).

${ }^{37}$ P. W. Brouwer and C. W. J. Beenakker, J. Math. Phys. 37, 4904 (1996).

${ }^{38}$ The equivalent equation of Ref. 14 as well as the subsequent discussion contain an error: the first power of $\gamma_{B}$ appears instead of $\gamma_{B}^{2}$.

${ }^{39}$ A. A. Golubov and M. Yu. Kupriyanov, Zh. Éksp. Teor. Fiz. 96, 1420 (1989) [Sov. Phys. JETP 69, 805 (1989)].

${ }^{40}$ A. V. Zaitsev, Phys. Lett. A 194, 315 (1994).

${ }^{41}$ A. V. Zaitsev, Zh. Éksp. Teor. Fiz. 86, 1742 (1984) [Sov. Phys. JETP 59, 1015 (1984)]. 